\title{
Amplification of electromagnetic radiation in a superlattice placed in a tilted magnetic field
}

\author{
M. A. Pyataev ${ }^{1}$, A. V. Shorokhov ${ }^{1}$, N. N. Khvastunov ${ }^{2}$, K. R. Vlasov ${ }^{1}$, V. D. Krevchik ${ }^{3}$, \\ M. B. Semenov ${ }^{3}$, K. N. Alekseev ${ }^{4}$, F. V. Kusmartsev ${ }^{4}$ \\ ${ }^{1}$ National Research Mordovia State University, Bolshevistskaya, 68, Saransk, 430005, Russia \\ ${ }^{2}$ Mordovian State Pedagogical Institute, Studencheskaya, 11 A, Saransk, 430007, Russia \\ ${ }^{3}$ Penza State University, Krasnaya, 40, Penza, 440026, Russia \\ ${ }^{4}$ Loughborough University, Loughborough, Leicestershire LE11 3TU, UK
}

pyataevma@gmail.com

\begin{abstract}
The interaction of electrons in a superlattice with electromagnetic radiation in presence of static electric and magnetic fields is investigated. The electric field is directed along the superlattice axis while the magnetic field is inclined at an arbitrary angle to the axis of superlattice. It is shown that the dependence of current in the superlattice on electric field in the general case can have several maxima. In some regions of electric and magnetic field values, the absorption coefficient for high frequency electromagnetic radiation can be negative that means the electromagnetic wave will be amplified. We note that negative absorption in the system is possible at some conditions at the region of positive differential conductivity in contrast to classical Bloch oscillator in which amplification takes place in case of negative differential conductivity only. This phenomenon can be used for the design of a teraherz amplifier and generator based on the superlattice.
\end{abstract}

Keywords: semiconductor superlattice, magnetic field, teraherz radiation.

Received: 6 November 2017

Revised: 13 November 2017

\section{Introduction}

Semiconductor superlattices are considered promising systems for creation of a compact teraherz radiation amplifier and generator. The idea of using Bloch oscillations in superlattices for the generation of the highfrequency electromagnetic radiation was proposed by Esaki and Tsu [1] more than forty years ago. However, the attempts to create a high-frequency generator based on the phenomenon of Bloch oscillations were not successful. It was shown later that the generation is suppressed by instabilities of space-charge waves in the superlattice in the regime of negative static differential conductivity (SDC). We note that in the case of classical Bloch oscillator the radiation amplification is possible only in the regime of negative SDC. One possible way to overcome this difficulty is the application of a magnetic field. Electron transport in superlattices placed in parallel $[2,3]$ or perpendicular [4-13] magnetic fields was studied previously. In particular, it was shown recently $[15,16]$ that the magnetic field applied to the superlattice in a direction perpendicular to the axis can shift the maximum on the static current-voltage characteristics (IV curve) towards the region of higher electric field. At the same time, the absorption coefficient can be negative for high frequencies at electric fields corresponding to positive SDC. That makes possible to achieve amplification of radiation without formation of space-charge wave instabilities. If the superlattice is placed in a tilted magnetic field, then the ballistic electron dynamics becomes more complicated and exhibits chaotic behavior [17-20]. This chaos manifests itself with the appearance of bifurcation points in the parameter space of the system. Various problems associated with the electron transport in the tilted magnetic field have been studied in the last decade [21-23]. The purpose of the present paper is to investigate the possibility of amplification of weak electromagnetic signal by the superlattice placed in both parallel electric and tilted magnetic fields. For this reason, we study absorption of electromagnetic radiation and its relation to the static IV curve.

\section{Current and absorption}

The schematic view of the investigated system is represented in Fig. 1. The direction of electric field which coincides with the axis of superlattice growth is denoted as $x$-axis and the magnetic field is inclined on angle $\theta$ to the electric field in $x z$-plane. In addition to constant electric field $E_{d c}$, we consider small perturbation oscillating with frequency $\omega$ and amplitude $E_{\omega}$. The total electric field is described by the equation:

$$
E_{x}(t)=E_{d c}+E_{\omega} \cos (\omega t) .
$$




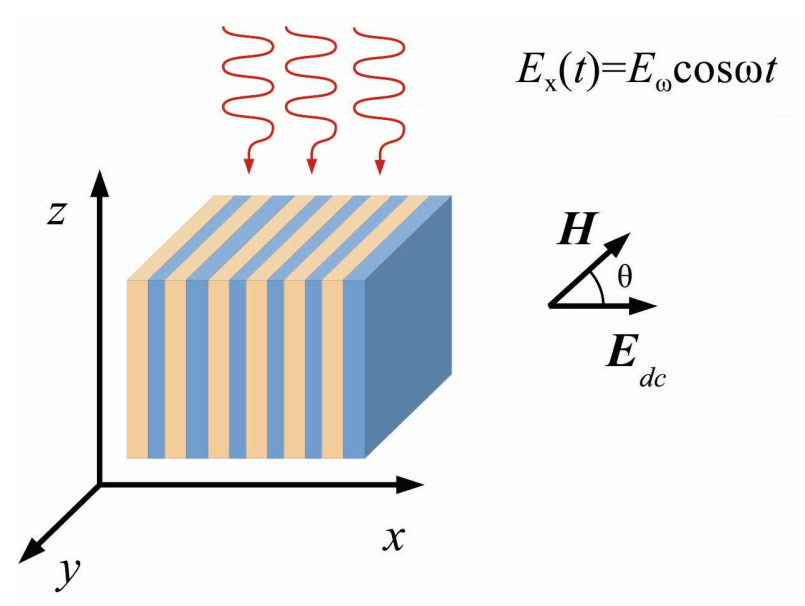

FIG. 1. Schematic representation of the system.

Our main goal is to determine the current produced by this field.

To find the current in the system, we use the semiclassical approximation which is based on the Boltzmann equation for electrons in external fields [15]. The superlatice properties in this approach are taken into account by the miniband dispersion relation for the electrons. In the relaxation-time approximation, the Boltzmann equation can be written in the form:

$$
\frac{\partial f}{\partial t}+\frac{1}{\hbar}\left(e \mathbf{E}(t)+\frac{e}{c} \mathbf{v} \times \mathbf{B}\right) \frac{\partial f}{\partial \mathbf{k}}=-\frac{f-f_{e q}}{\tau},
$$

where $\mathbf{v}$ is the electron velocity, $\tau$ is the relaxation time, $\mathbf{k}$ is the quasi-wave vector, and $f_{e q}(\mathbf{k})$ is the equilibrium distribution function. In the present study, we consider the case of spatially uniform fields and electron distribution.

The dependence of electron energy on quasi-wave vector in the superlattice is described by the well-known equation from tight-binding approximation:

$$
\varepsilon(\mathbf{k})=-\frac{\Delta}{2} \cos \left(k_{x} d\right)+\frac{\hbar^{2}\left(k_{y}^{2}+k_{z}^{2}\right)}{2 m},
$$

where $d$ is the superlattice period and $\Delta$ is the miniband width.

Based on the method of characteristics, we can represent the distribution function in terms of solutions for ballistic equations of motion for the electrons in the external fields [24]. The equation for quasi-wave vector evolution at the ballistic electron motion has the form:

$$
\hbar \dot{\mathbf{k}}=e \mathbf{E}(t)+\frac{e}{c} \mathbf{v}(\mathbf{k}) \times \mathbf{B}
$$

Let $\mathbf{k}^{*}\left(t, t_{0}\right)$ denotes the solution of Eq. (4) corresponding to the initial condition $\mathbf{k}\left(t_{0}\right)=\mathbf{k}_{0}$. If we substitute $\mathbf{k}^{*}$ into Eq. (2) then its left-hand side becomes a total derivative of $f$ with respect to $t$ :

$$
\frac{d f\left(\mathbf{k}^{*}, t\right)}{d t}=-\frac{f\left(\mathbf{k}^{*}, t\right)-f_{e q}\left(\mathbf{k}^{*}\right)}{\tau} .
$$

Then, the steady-state solution of Eq. (2) can be represented in the form:

$$
f(\mathbf{k}, t)=\frac{1}{\tau} \int_{-\infty}^{t} d t_{0} f_{e q}\left(\mathbf{k}^{*}\left(t, t_{0}\right)\right) e^{-\frac{t-t_{0}}{\tau}},
$$

where $\mathbf{k}^{*}\left(t, t_{0}\right)$ is the solution of Eq. (4) satisfying the condition $\mathbf{k}^{*}\left(t, t_{0}\right)=\mathbf{k}$ for every $t_{0}$.

The density $j$ of current in $x$-direction can be represented in terms of the distribution function $f$ by the following equation:

$$
j(t)=\frac{2 e}{(2 \pi)^{3}} \int d^{3} \mathbf{k} f(\mathbf{k}, t) v_{x}(\mathbf{k})
$$

where $v_{x}=\frac{1}{\hbar} \frac{\partial \varepsilon}{\partial k_{x}}$ is $x$-component of the electron velocity, factor $(2 \pi)^{3}$ in the denominator takes into account density of states and factor 2 appears in the numerator for spin. As it follows from Eq. (3) the velocity $v_{x}$ is given 
by:

$$
v_{x}\left(k_{x}\right)=\frac{\Delta d}{2 \hbar} \sin \left(k_{x} d\right)
$$

Now combining Eqs. (6) and (7) we obtain the following equation for the current:

$$
j(t)=\frac{e}{4 \pi^{3} \tau} \int d^{3} \mathbf{k} f_{e q}(\mathbf{k}) \int_{-\infty}^{t} d t_{0} v_{x}\left(\mathbf{k}^{*}\left(t, t_{0}\right)\right) e^{-\frac{t-t_{0}}{\tau}} .
$$

In the present study, we consider the case of low temperatures and low electron concentrations $N$. In this case almost all electron quasi-wave vectors are concentrated at the bottom of miniband near the point $\mathbf{k}=0$. The equilibrium distribution function in this approximation can be represented as follows [?]:

$$
f_{e q} \approx 4 N \pi^{3} \delta(\mathbf{k})
$$

where $\delta(x)$ is the Dirac $\delta$-function.

Let us introduce the dimensionless components of the quasi-wave vector: $K_{x}=k_{x} d, K_{y}=k_{y} d \sqrt{m / m_{x}}$, and $K_{z}=k_{z} d \sqrt{m / m_{x}}$, where $m$ is electron effective mass in perpendicular direction and $m_{x}=2 \hbar / d^{2} \Delta$ is the electron mass in the parallel direction. Then the ballistic equations of motion can be rewritten as follows:

$$
\begin{aligned}
\dot{K}_{x} & =\omega_{B}+\omega_{\perp} K_{y}+\beta \sin (\omega t), \\
\dot{K}_{y} & =-\omega_{\perp} \sin K_{x}+\omega_{\|} K_{z}, \\
\dot{K}_{z} & =-\omega_{\|} K_{y}
\end{aligned}
$$

where $\omega_{B}=e d E_{d c} / \hbar$ is the Bloch frequency, $\omega_{c}=e B / m c$ is the cyclotron frequency, $\omega_{\perp}=\omega_{c} \sin \theta, \omega_{\|}=$ $\omega_{c} \cos \theta$ and $\beta=e E_{\omega} d / \hbar$ is the renormalized amplitude of the high-frequency electric field.

To understand various possibilities of quasi-wave vector dynamics from equations (11), (12) and (13), we consider first a few simplified cases. One can see from equations (11) that the parallel magnetic field $\left(\omega_{\perp}=0\right)$ does not affect the $x$ component of the quasi-wave vector. In that case, the IV curve of the system has the form obtained by Esaki and Tsu [1].

If the magnetic field is directed parallel to $z$-axis, then the $y$ coordinate can be separated from others and the system of equations (11)-(13) is decomposed. In this case, the equation for $\dot{K}_{x}$ can be transformed into the equation of motion for unharmonic pendulum:

$$
\ddot{K}_{x}+\omega_{\perp} \sin K_{x}=\beta \sin (\omega t)
$$

The constant electric field $E_{d c}$ is included into the equations of motions through initial condition:

$$
\dot{K}_{x}\left(t_{0}\right)=\omega_{B}
$$

As follows from Eq. (14), in the absence of perturbation $(\beta=0)$, there are two regimes of motion for electrons depending on the initial conditions: cyclotron-like oscillations and Bloch type oscillations [4]. In the cyclotron-like regime, the electron quasi-wave vector does not reach the boundary of the first Brillouin zone while in the regime of Bloch type oscillations the quasi-wave vector increases infinitely. If the initial electron quasi-wave vector is zero, then the system undergoes a transition from cyclotron-like oscillations to Bloch type oscillations at the electric field corresponding to condition $\omega_{B}=2 \omega_{c}$. In the general case of the inclined magnetic field, there are several bifurcation points and the analytical investigation of the ballistic equations of motion becomes very complicated. In the present study, we have performed numerical solution of the system (11)-(13) and found the dependence of electron quasi-wave vector on time. Then, by equation (9), we have found the time-dependent current in the steady-state regime. After that, we have calculated the absorption as the average dissipation power $P$ in a unit volume by the following equation:

$$
P=\frac{1}{T} \int_{0}^{T} j(t) E(t) d t
$$

where $T=2 \pi / \omega$ is the period of the small external oscillating field. 


\section{Results and discussion}

Let us consider first the dependence of current on voltage for the case of time independent fields $\left(E_{\omega}=0\right)$. We note that the equations of motion in this case are similar to the equation of motion for an anharmonic pendulum in the field of a plane wave which is the well-known example of a system with chaotic behavior $[17,18]$

$$
\ddot{K}_{z}+\omega_{\|}^{2} K_{z}=-\omega_{\|} \omega_{\perp} \sin \left(\frac{\omega_{\perp}}{\omega_{\|}} K_{z}-\omega_{B} t+\varphi\right) .
$$

However, as it follows from Eq. (9), to obtain the current in a dissipative system, one needs to know the values of qusimomentum for a finite period of time (about a few $\tau$ ) while the chaotic dynamics manifests itself in a very long time. That is why the chaotic dynamics of the systems does not lead to a chaotic behavior of the IV characteristics or absorption. However, bifurcation between different regimes of motion can lead to appearance of a very sharp changes in IV curve.
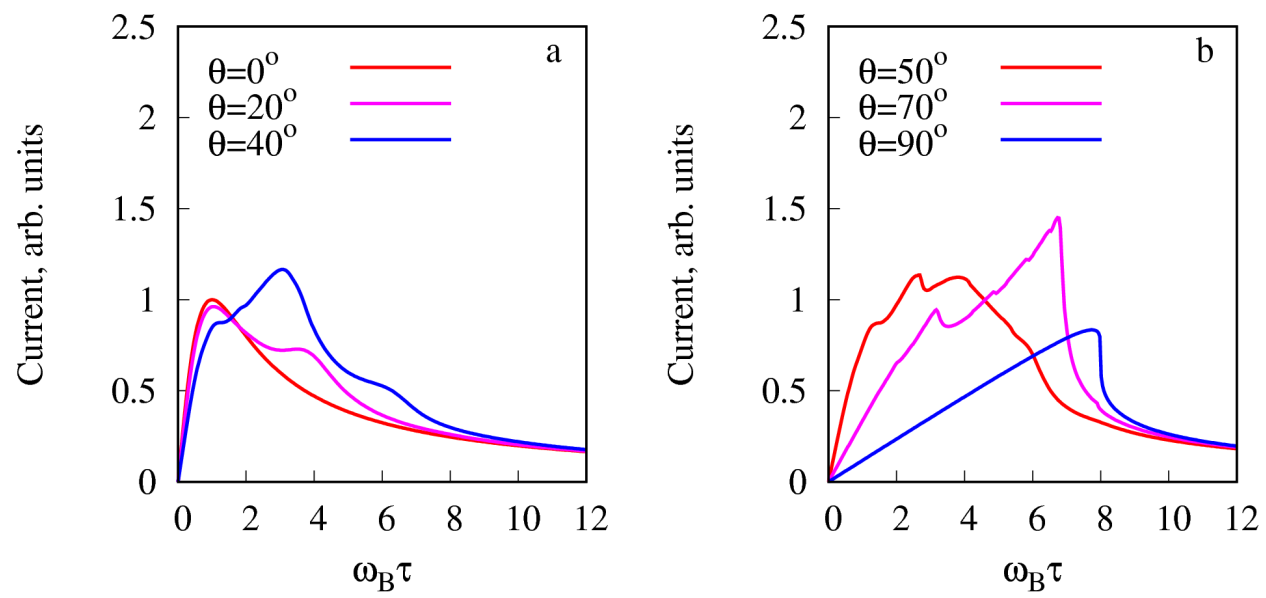

FIG. 2. The dependence of current on the Bloch frequency for various values of angle $\theta$ at $\omega_{c} \tau=4$.

The dependence of current on the electric field for different inclination angles $\theta$ is shown in Fig. 2. One can see from Fig. 2 that the current as a function of electric field has one maximum in the cases of parallel and perpendicular magnetic field. The decrease on the IV curve corresponds to the transition of the transport regime to the Bloch type oscillations. The value of $\omega_{B}$ corresponding to the transition is determined by relation $\omega_{B} \tau=1$ in the case of $\theta=0$ and is close to the value $\omega_{B}=2 \omega_{c}$ in the case of $\theta=90^{\circ}$. One can see that with increase in angle $\theta$, the position of global maximum on the IV curve is shifted to the region of higher electric fields. Additional peaks occur on the curve in the case of tilted magnetic field due to the appearance of additional bifurcation points in the parameter space. In particular, there is one extra maximum of current at relatively small values of $\theta$ (about $20^{\circ}$ ). Our analysis shows that at electric fields corresponding to the second increase of current, the negative absorption of weak electromagnetic radiation can be obtained. The dependence of absorption coefficient on frequency for this case is shown in Fig. 3. The negative absorption in this case occurs in the region of positive static differential conductivity. Similar results can be obtained in the case of large $\theta$. The dependence of absorption on frequency for $\theta=80^{\circ}$ is shown in Fig. 4 . One can see from Fig. 4 that the negative absorption at positive differential conductivity can be achieved in the region of electric fields between two local maximums on the IV curve.

\section{Conclusion}

We have investigated a static current-voltage characteristics and absorption of high-frequency electromagnetic radiation in a semiconductor superlattice in the presence of a parallel electric field and tilted magnetic field. In the cases of parallel and perpendicular magnetic field, the IV curve of the system has one maximum. The positions of maximum on the dependence of current on the Bloch frequency are determined by inverse relaxation time in the parallel field and by doubled cyclotron frequency in perpendicular field. We have shown in the present study that the inclined magnetic field applied to a superlattice can lead to appearance of additional maximums and minimums on the static IV curve. Near the local maximums of the IV curve there are regions of field values in which the static differential conductivity is positive, but the high-frequency absorption coefficient is negative. In this case, the 


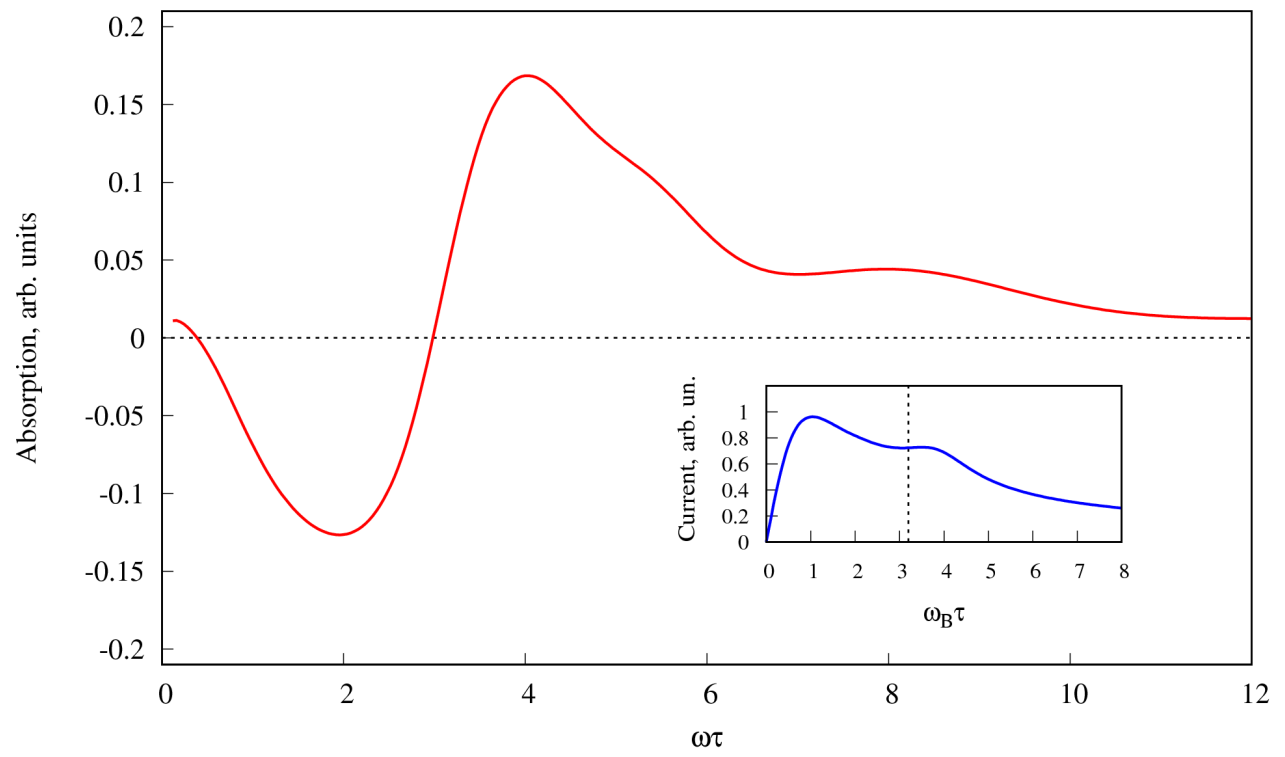

FIG. 3. The dependence of absorption on frequency $\omega$ at $\theta=20^{\circ}, \omega_{c} \tau=4$ and $\omega_{B} \tau=3.2$. The inset shows the dependence of current on the Bloch frequency (IV curve) for the same parameters. The vertical dashed line marks the Bloch frequency $\omega_{B}=3.2 / \tau$ which was used for calculation of the absorption. One can see that static differential conductivity is positive at this value of $\omega_{B}$.

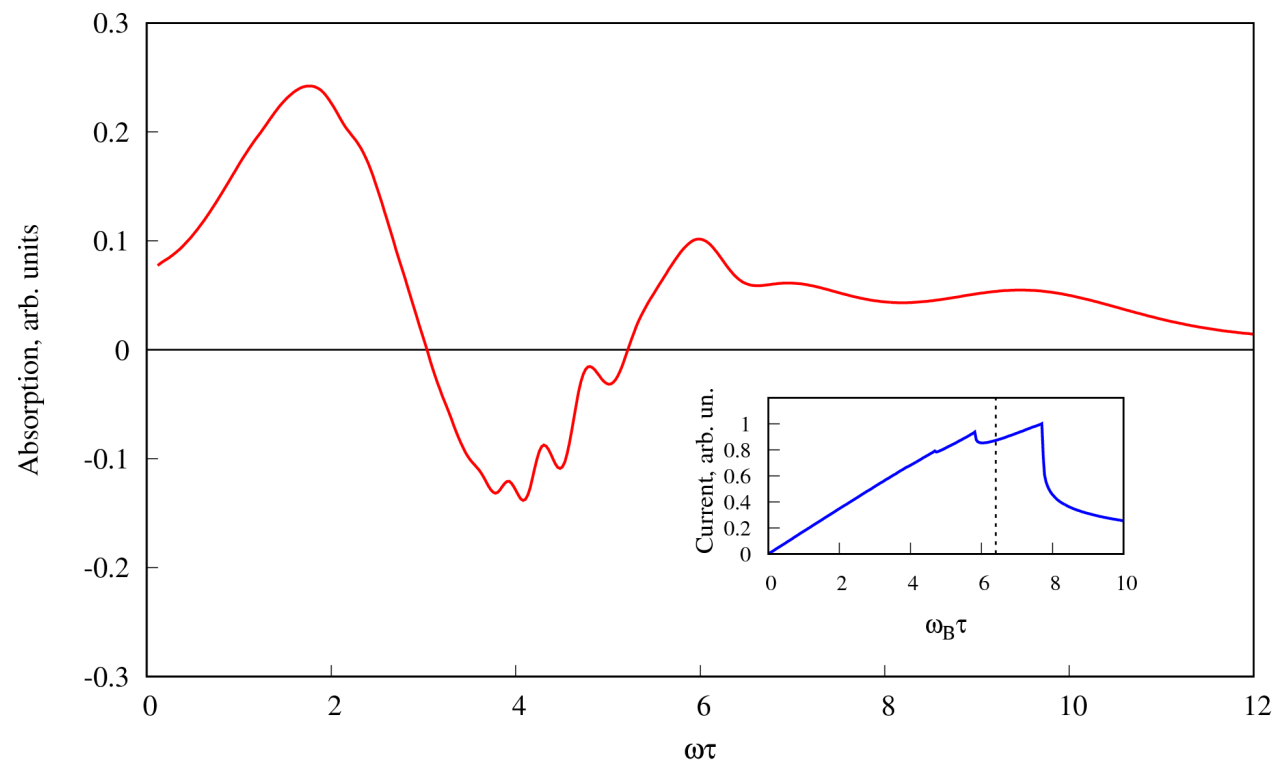

FIG. 4. The dependence of absorption on frequency $\omega$ at $\theta=80^{\circ}, \omega_{c} \tau=4$ and $\omega_{B} \tau=6.4$. The inset shows the dependence of current on the Bloch frequency (IV curve) for the same parameters. The vertical dashed line marks the Bloch frequency $\omega_{B}=6.4 / \tau$ which was used for calculation of the absorption. One can see that static differential conductivity is positive at this value of $\omega_{B}$. 
high-frequency radiation can be amplified by the superlattice. At the same time, charge wave instabilities should not appear because of positive SDC. We note that the frequency of radiation which can be generated by the system in this regime is determined by parameters of the superlattice and the value of magnetic field. Our estimations show that for a GaAs $/ \mathrm{Al}_{x} \mathrm{Ga}_{1-x}$ As superlattice with the period $d=6 \mathrm{~nm}$, miniband width $\Delta=60 \mathrm{meV}$ and magnetic field $B=2.5 \mathrm{~T}$ the generated frequency is about $1 \mathrm{THz}$. Hence, such fields configuration can be used in designing of a terahertz generator based on the semiconductor superlattice.

\section{Acknowledgements}

This work was supported by the Ministry of Education and Science of the Russian Federation (project no. 3.6321.2017/8.9), and RFBR (project no. 17-02-00969).

\section{References}

[1] Esaki L., Tsu R. Superlattice and negative differential conductivity in semiconductors. IBM J. Res. Dev., 1970,14, P. 61.

[2] Hummel A.B., Bauer T., et al. Influence of a strong magnetic field on the Wannier-Stark states of an electrically biased $\mathrm{GaAs}_{\mathrm{Al}} \mathrm{Al}_{x} \mathrm{Ga}_{1-x} \mathrm{As}$ superlattice. Phys. Rev. B, 2003, 67, 045319-1-10.

[3] Patane A., Mori N., et al. Magnetic-field-induced suppression of electronic conduction in a superlattice. Phys. Rev. Lett., 2004, 93, 146801-1-4.

[4] Bass F.G., Tetervov A.P. High-frequency phenomena in semiconductor superlattices. Phys. Rep., 1986, 140 (5), P. $237-322$.

[5] Polyanovskii V.M. Nonlinear conductivity of a semiconductor with a superlattice in a strong magnetic field. Sov. Phys. Semicond., 1980, 14, P. 718-720.

[6] Bass F.G., Lykakh V.A., Tetervov A.P. Cyclotron resonace in superlattice semiconductor. Sov. Phys. Semicond., 1980,14, P. $1372-1376$.

[7] Bass F.G., Zorchenko V.V., Shashora V.I. To the theory of galvanomagnetic and high-frequency phenomena in semiconductors with a superlattice. Sov. Phys. Semicond., 1981, 15, P. 263-270.

[8] Sibille A., Palmier J.F., et al. Semi-classical magnetotransport in a superlattice miniband. Europhys. Lett., 1990 , 13 (3), P. $279-284$.

[9] Miller D., Laikhtman B. Classical theory of the perpendicular magnetoresistance in superlattice. Phys. Rev. B, 1995, 52, P. 12191-12201.

[10] Elsholz F., Wacker A., et al. Magnetotransport through semiconductor superlattices. Phys. Rev. B, 2001, 63, 033312-1-4.

[11] Feil T., Gerl C., Wegscheider W. Transport properties of a shunted surface superlattice in an external magnetic field. Phys. Rev. B, 2006, 73, 125301-1-7.

[12] Kosevich Yu.A. Anomalous Hall velocity, transient weak supercurrent, and coherent Meissner effect in semiconductor superlattices. Phys. Rev. B, 2001, 63, 205313-1-19.

[13] Bauer T., Kolb J., et al. Coherent Hall effect in a semiconductor superlattice. Phys. Rev. Lett., 2002, 88, 086801-1-4.

[14] Hummel A.B., Blöser C., et al. Electro-optic investigation of the coherent Hall effect in semiconductor superlattices. Phys. Stat. Sol. (b), 2005, 242 (6), P. 1175-1178.

[15] Hyart T., Mattas J., Alekseev K.N. Model of the influence of an external magnetic field on the gain of terahertz radiation from semiconductor superlattices. Phys. Rev. Lett., 2009, 103, 117401-1-4.

[16] Shorokhov A.V., Pyataev M.A., et al. Physical principles of the amplification of electromagnetic radiation due to negative electron masses in a semiconductor superlattice. JETP Lett., 2014, 100 (12), P. 766-770.

[17] Fromhold T.M., Krokhin A.A., et al. Effects of stochastic webs on chaotic electron transport in semiconductor superlattices. Phys. Rev. Lett., 2001, 87, 046803-1-4.

[18] Balanov A.G., Fowler D., et al. Bifurcations and chaos in semiconductor superlattices with a tilted magnetic field. Phys. Rev. E, 2008, 77, 026209-1-13.

[19] Selskii A.O., Koronovskii A.A., et al. Studying transitions between different regimes of current oscillations generated in a semiconductor superlattice in the presence of a tilted magnetic field at various temperatures. Tech. Phys. Lett., 2015, 41 (8), P. 768-770.

[20] Selskii A.O., Hramov A.E., et al. Bifurcation phenomena in a semiconductor superlattice subject to a tilted magnetic field. Phys. Lett. A, 2016, 380, P. 98-105.

[21] Balanov A.G., Greenaway M.T., et al. The effect of temperature on the nonlinear dynamics of charge in a semiconductor superlattice in the presence of a magnetic field. J. Exp. Theor. Phys., 2012, 114 (5), P. 836-840.

[22] Alexeeva N., Greenaway M.T., et al. Controlling high-frequency collective electron dynamics via single-particle complexity. Phys. Rev. Lett., 2012, 109, 024102-1-5.

[23] Balanov A.G., Koronovskii A.A., et al. Space charge dynamics in a semiconductor superlattice affected by titled magnetic field and heating. Physics of Wave Phenomena, 2016, 24 (2), P. 103-107.

[24] Budd J. Generalized path variable method. J. Phys. Soc. Japan, 1963, 18, P. 142. 